\title{
Análisis de las Fracturas Mandibulares Causadas por Accidentes Laborales. Estudio Descriptivo Retrospectivo
}

\author{
Analysis of Mandibular Fractures Caused by Work Related Accidents. \\ Retrospective Descriptive Study
}

Gonzalez Mora, L. E.*; Vargas Farren, I.*; Pedemonte Trewhela, C.*; Canales Trkovic, M.* \& Sáez Salas, F.* \& Verdugo-Avello, F."

GONZALEZ, M. L. E.; VARGAS, F. I.; PEDEMONTE, T. C.; CANALES, T. M. \& SÁEZ, S. F. \& VERDUGO-AVELLO, F. Análisis de las fracturas mandibulares causadas por accidentes laborales. Estudio descriptivo retrospectivo. Int. J. Odontostomat., 9(2):198-203, 2015.

RESUMEN: El objetivo de este estudio fue evaluar la tendencia de los pacientes con fracturas mandibulares asociados a accidentes laborales. Se realizó un estudio descriptivo retrospectivo en todos los casos de fracturas mandibulares que asistieron al servicio de cirugía maxilofacial del Hospital Clínico Mutual de Seguridad C.Ch.C., Santiago de Chile en un periodo de 4 años (enero 2009 - diciembre 2012). Todas las fracturas mandibulares fueron consignadas. La información fue recopilada a través de revisión de fichas clínicas electrónicas. Entre los años 2009 al 2012 se presentaron un total de 74 pacientes incluidos en el estudio con 102 rasgos de fracturas mandibulares. La fractura más común fue la de cóndilo mandibular (35 pacientes). Se observó una tasa de complicaciones del 8\%. Los resultados mostrados en este estudio están en línea con la literatura y el análisis de este reporte provee información para el diseño de planes de prevención de riesgos, especialmente para desarrollar medidas de protección facial. Nivel de Evidencia: Tipo II. Estudio descriptivo

PALABRAS CLAVE: fractura mandibular, accidente laboral, estudio retrospectivo.

\section{INTRODUCCIÓN}

La etiología de las fracturas mandibulares varia según la sociedad y la cultura estudiada (Fridrich et al., 1992; Bataineh, 1998; Boole et al., 2001; Lin et al., 2007; Bormann et al., 2009). En base a esto, reportes recientes muestran que daños al área maxilofacial son causados habitualmente por traumas, específicamente accidentes por motocicleta, asaltos y caídas (Ellis et al., 1985; Timoney et al., 1990; Vetter et al., 1991; Edwards et al., 1994; Hogg et al., 2000). Muy poco se sabe sobre la etiología de las fracturas faciales en la población chilena trabajadora cubiertos por la ley del trabajo.

Chile es un país que realiza un monitoreo anual de la tasa de accidentes laborales, lo que ha demostrado en los últimos 60 años la necesidad de implementar seguros para los trabajadores ante un eventual accidente traumático ligado al trabajo. Estos seguros desarrollaron centros de trauma para tratar las complejas patologías agudas y crónicas resultantes del trabajo, como es el caso de nuestra institución Bataineh). Durante el año 2012 en Chile, 4,4 millones de trabajadores estuvieron cubiertos contra alguna patología derivada del trabajo. Aproximadamente el $50 \%$ de éstos están cubiertos por nuestro centro hospitalario. El $7 \%$ del total de los pacientes ingresados a nuestro hospital (centro de referencia de Trauma nivel I), son referidos al Servicio de Cirugía Oral y Maxilofacial por daños a nivel dental y facial.

El presente estudio tiene como objetivo evaluar el perfil epidemiológico y las características del tratamiento, específicamente de los pacientes con fracturas mandibulares asociados a accidentes laborales que ingresaron al Hospital Clínico Mutual de Seguridad C.Ch.C.

\footnotetext{
* Unidad de Cirugía Maxilofacial, Policlínico de Especialidades, Hospital Clínico Mutual de Seguridad C.Ch.C., Santiago, Chile.

" Cátedra y Clínica de Cirugía. Facultad de Odontología, Sede Concepción. Universidad Andrés Bello, Chile.
} 


\section{MATERIAL Y MÉTODO}

Se realizó un estudio descriptivo retrospectivo en todos los casos de fracturas mandibulares que asistieron al servicio de cirugía maxilofacial del "Hospital Clínico Mutual de Seguridad C.Ch.C.", Santiago de Chile. En un periodo de 4 años (enero 2009 - diciembre 2012). La información fue recopilada a través de revisión de fichas clínicas electrónicas de manera sistematizada por un investigador.

Como criterios de exclusión se establecieron los siguientes: información en ficha clínica y/o protocolo quirúrgico incompleto, pacientes ingresados con cirugía de urgencia realizada por otro centro hospitalario, accidentes que no fueran causados por el trabajo (trabajo o trayecto).

Las causas de las fracturas mandibulares fueron clasificadas en cinco categorías: caídas de altura, golpe con objeto (herramienta o material industrial), accidente de tráfico (motocicleta, automóvil, bicicleta y atropello), violencia y otros. Fueron incluidos todos los pacientes con fractura mandibular de uno o más rasgos, con tratamiento abierto y requerimiento de fijación interna funcional o reducción ortopédica. La locación mandibular fue dividida en sínfisis, parasínfisis, cuerpo, ángulo, rama, proceso coronoides, cóndilo y fracturas conminutas.

Fueron analizadas la frecuencia, tipo de fractura y causa del daño, edad de distribución y género, orden de tratamiento quirúrgico, así como también las complicaciones y secuelas. Las comparaciones fueron realizadas mediante la prueba chi cuadrado. Esto fue seguido por un análisis de regresión lo gística en caso de haber diferencias significativas entre las variables.

La secuencia de evaluación fue la siguiente: Una vez que el examen físico indica una fractura mandibular se realiza una tomografía computada cortes de 1-2 $\mathrm{mm}$ de la zona traumatizada.

Las indicaciones quirúrgicas establecidas en el servicio son fracturas que presenten un desplazamiento y/o movilidad de los bordes (inestabilidad de los fragmentos), con o sin alteración de la oclusión dentaria. En la mayoría de los casos se realizó primero la reducción y fijación del rasgo en la zona dentada para tratar después la zona fracturada edéntula.
Diagnosticado la fractura hay dos opciones; en la primera, se ingresa a pabellón con anestesia general para la reducción y fijación con placas y tornillos de osteosíntesis. Se realiza en conjunto un bloqueo intermaxilar con tornillos de bloqueo y pasando un alambre grueso 0,6 mm entre ambas arcadas de manera bilateral, en caso de dentición de mesa oclusal posterior completa. Una vez lograda máxima intercuspidación se realiza una reducción de los rasgos óseos de la fractura. Al quedar los rasgos de fractura en adecuada aproximación se realiza la fijación con elementos de osteosíntesis.

En los tratamientos abiertos de las fracturas de cuerpo y de la zona anterior mandibular, el tratamiento realizado es con doble placa, generalmente una de perfil mayor en la zona de compresión o margen basilar. En algunos casos especiales, en fracturas anteriores se usa el sistema "Lag Screw" (Fig. 1). En la zona del ángulo se realiza el sistema descrito por Champy en la cual se fija una placa a la línea oblicua externa en caso de menor desplazamiento y doble placa en caso de fracturas más desplazadas o complejas (Fig. 2). El cóndilo mandibular se trata quirúrgicamente mediante reducción y fijación con: doble placa, placa en " $Y$ " o placa tridimensional (Fig. 3). Como objetivo de la fijación interna funcional se plantea devolver la anatomía perdida y devolver la funcionalidad del sistema estomatognático.

En cuanto al tratamiento ortopédico, solo se indicó en los casos de fracturas condilares intracapsulares o si el paciente rechazó la opción de tratamiento abierto. En primer lugar se lleva a cabo un bloqueo intermaxilar con elásticos por una semana. Posteriormente se realiza un tratamiento kinesiológico mediante movimientos de apertura, protrusión, lateralidades 5 veces al día, durante un periodo de 3 semanas variando según su evolución clínica.

Comúnmente se realiza un abordaje o acceso intraoral, dejando el retromandibular transparotideo y/ o preauricular para las fracturas condilares. En algunos casos se puede utilizar la herida asociada a la zona traumatizada.

Se realiza una tomografía computadorizada postoperatoria para evaluar la posición de los rasgos, y controles periódicos a los 7, 15, 30 días y 3 meses con evaluación clínica de los resultados mediante examen clínico de función mandibular.

La aprobación del comité de ética hospitalario fue requerida y aceptada. 


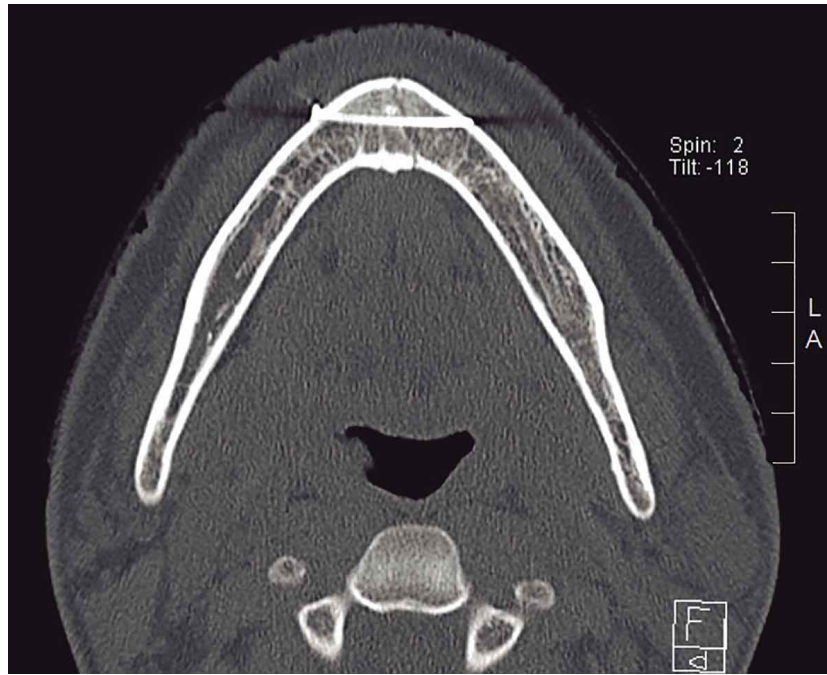

Fig. 1. Tomografía computadorizada (TC) en corte axial donde se observa fractura parasinfisiaria mandibular tratada con sistema "Lag Screw" donde el tornillo pasa perpendicular al rasgo de fractura.

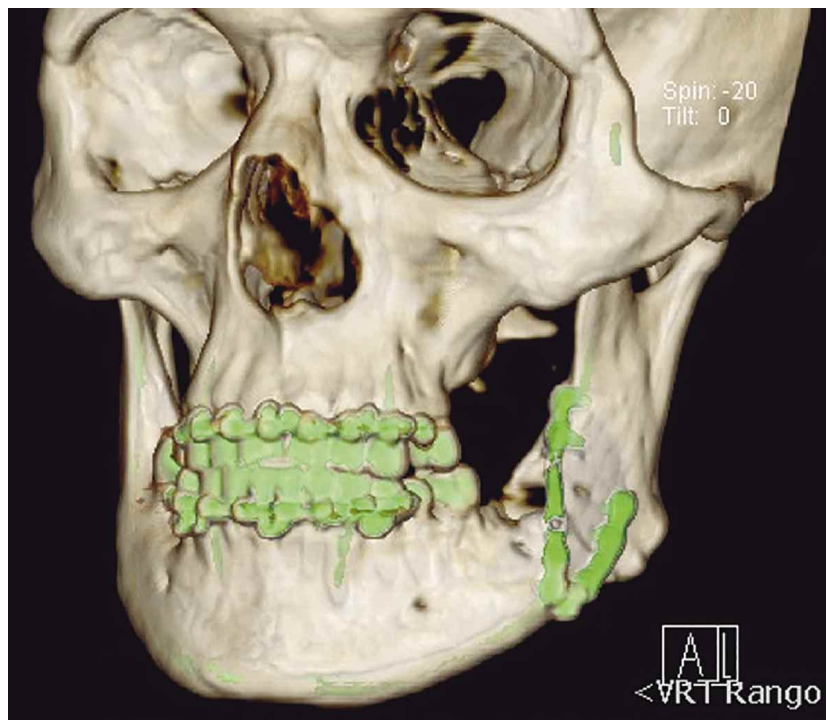

Fig.2. Fractura de ángulo mandibular tratada mediante doble placa.

\section{RESULTADOS}

Entre los años 2009 al 2012 se presentaron en la unidad de cirugía maxilofacial del Hospital Mutual de Seguridad un total de 74 pacientes incluidos en el estudio con 102 rasgos de fracturas mandibulares.

La muestra se compuso en su mayoría de hombres, 65 pacientes $(86,5 \%)$ y solo 9 mujeres $(13,5 \%)$, con una relación mujer: hombre de 1:7,2. El promedio

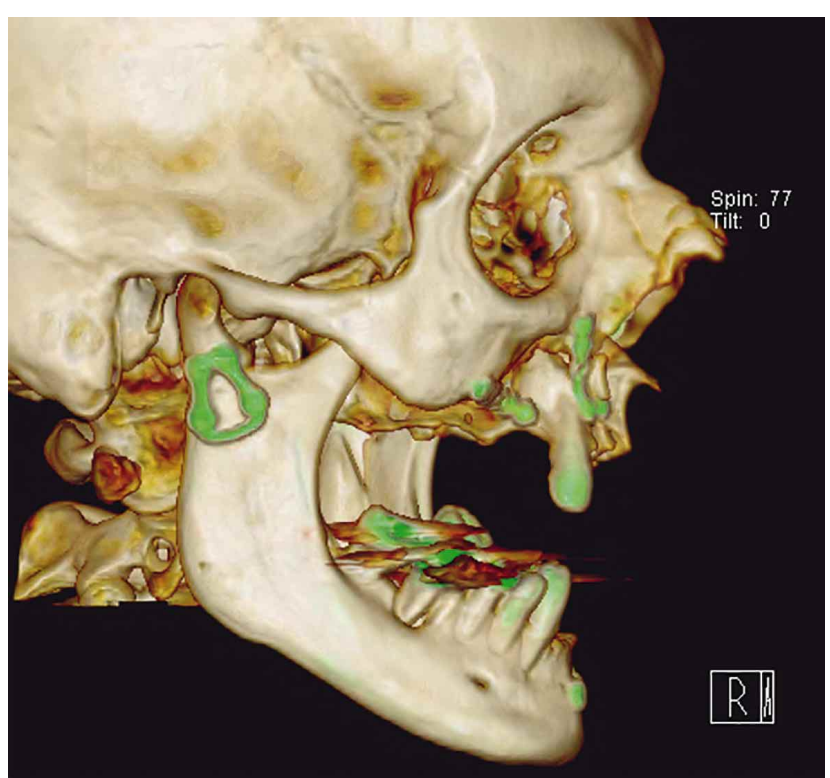

Fig. 3. Fractura de cóndilo mandibular tratada mediante oseosíntesis tridimensional

de edad fue de 39 años, en un rango de edad desde los 19 años hasta los 83 , con un predominio en el rango de edad de los 19 a los 29 y 30 a los 39 años (43 pacientes).

Veintisiete pacientes $(36,5 \%)$ sufrieron accidentes de tránsito, 16 pacientes sufrieron algún tipo de agresión, 15 pacientes resultaron de golpe con objeto. De los accidentes de tránsito predominaron los accidentes automovilísticos. Nueve pacientes tuvieron una caída de altura (Fig. 4)

\section{Causa de accidente}

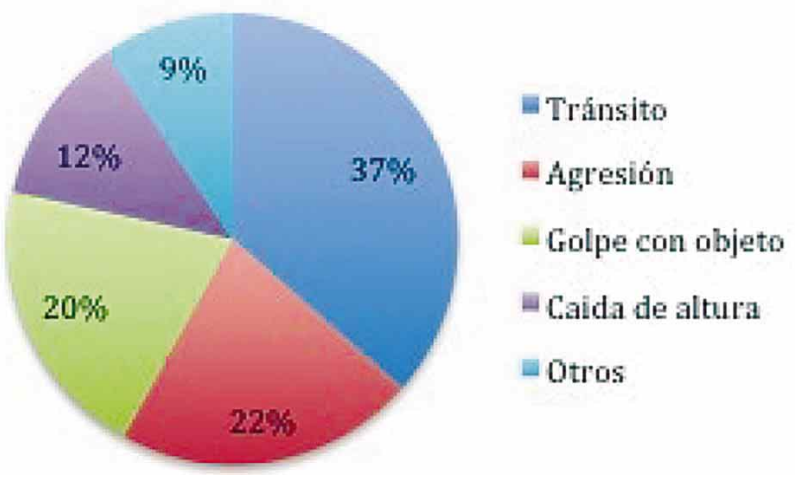

Fig. 4. Causas de las fracturas mandibulares en accidentes laborales. 
Entre los 102 rasgos de fractura se encontró como la localización con mayor frecuencia la de cóndilo mandibular, seguido de paramediana y cuerpo. El proceso coronoides se vio afectado en una oportunidad siendo las menos común (Tabla I).

Tabla I. Distribución de los rasgos de fractura según localización.

\begin{tabular}{lc}
\hline Localización & $\begin{array}{c}\text { Número de } \\
\text { rasgos }\end{array}$ \\
\hline Sinfisiaria & 8 \\
Paramediana & 22 \\
Cuerpo & 19 \\
Ángulo & 12 \\
Rama & 5 \\
Cóndilo & 35 \\
Coronoides & 1 \\
\hline
\end{tabular}

Hubo 22 pacientes con fracturas múltiples mandibulares, 16 pacientes con dos rasgos de fractura y 6 con triple rasgo. La combinación más frecuente fue la de paramediana con cóndilo. La combinación de rasgos no tubo diferencias significativas con la tasa de complicaciones.

El $75 \%$ de los pacientes fue sometido a reducción y fijación con elementos de osteosíntesis. El cuarto restante, compuesto por fracturas de cóndilo mandibular, fue tratado mediante tratamiento ortopédico y kinésico. Hubo 2 pacientes con fracturas bicondileas intracapsulares que fueron tratados también kinésicamente.

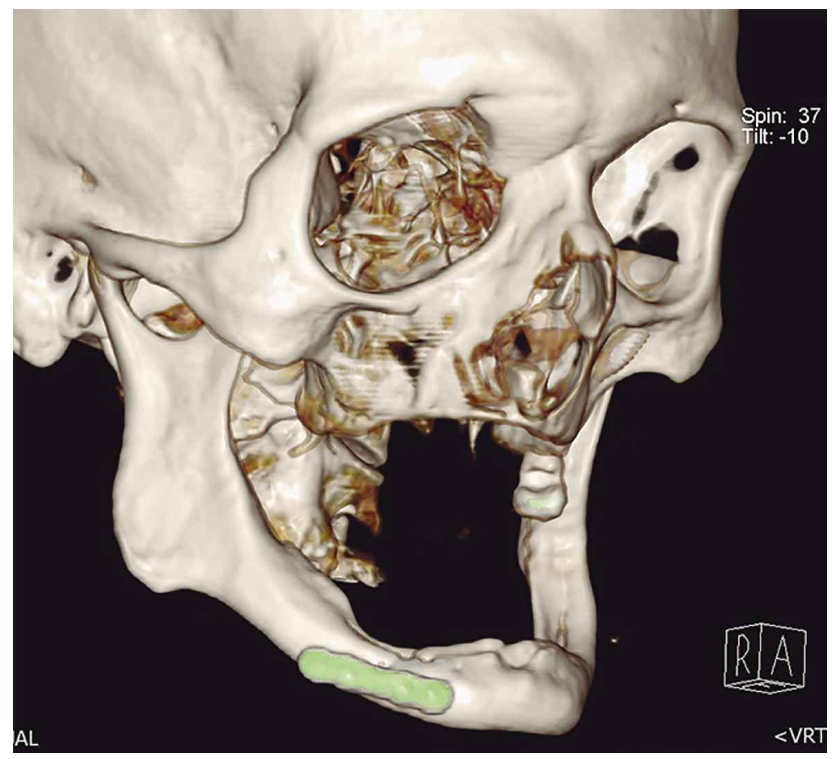

Fig. 5. Fijación de única placa 0.8 sistema Matrix Mandibular Synthes $®$ en mandíbula atrófica.
Se registraron 20 "fracturas múltiples" con una tendencia a tratar primero o de forma única al área dentada. En 10 pacientes solo se trato el área con presencia de oclusión, dejando al cóndilo con tratamiento ortopédico. En los 10 restantes se trató el área dentada, con excepción de solo un caso donde fue tratado primero el cóndilo. Es decir, dejando en segunda instancia el cóndilo o ángulo mandibular en la mayoría de los casos.

Se contabilizaron 6 complicaciones (tasa 8\%); 2 alteraciones oclusales, 2 disfunciones de articulación temporomandibular y 2 infecciones. No hubo complicaciones asociadas a las "fracturas múltiples" tratadas. No se encontró relación entre las variables.

\section{DISCUSIÓN}

Chile es un país con una tasa de accidente laboral de 4,4 por cada 100 trabajadores (año 2012) siendo de un 7,3 hace 10 años. Esta mejora en la tasa fue generada, entre otros, gracias a que se estableció la necesidad de recolectar evidencia en los últimos 60 años para implementar programas de prevención para los trabajadores (Bataineh). Nuestro hospital sólo asegura trabajadores adultos, lo que implica que los niños, estudiantes y dueñas de casa, así como también los adultos mayores de 65 años o más no estén cubiertos por esta ley. Sin embargo, la ley del accidente del trabajo permite una identificación de una población adulta variada con un número de casos relativamente alto.

La mandíbula es uno de los huesos faciales más resistentes de la cara, pero esta sujeto a fracturas por algunas razones ya descritas en la literatura: es el parachoques natural de la cara, es un arco abierto, componiendo el tercio inferior facial siendo más propenso a ser el primer impacto en accidentes de tránsito y peleas. Además sufre al igual que todos los otros huesos una atrofia progresiva con la edad y perdida dentaria (Fig. 5) (Girotto et al., 2001).

Este estudio describe la epidemiología de 74 pacientes con fracturas mandibulares como consecuencia de un accidente laboral durante un periodo de 4 años. Los relación hombre mujer fue de 7:1. La predominancia del género masculino en esta población de pacientes es un hallazgo constante en la mayoría de los estudios. Sin embargo, esta proporción fue mayor de lo que se señala en otros países (Gamo- 
nal et al., 2010; Lamphier et al., 2003). La población de nuestro estudio tiende a ser mayor en edad que otros reportes, probablemente porque los menores de 18 años están excluidos del promedio.

El rango de edad más afectado el de los 20 a los 29 años junto con la década siguiente (30 a 39 años), lo cual coincide con otros reportes epidemiológicos (Patrocínio et al., 2005). Puede ser una causa el mayor desplazamiento que requiere el trayecto laboral en una ciudad con alto tráfico y la falta de uso de dispositivos de seguridad tanto en los vehículos motorizados como en las labores de construcción. En personas que por su edad pueden pasar más horas trasladándose y haciendo tareas que requieren mayor esfuerzo físico por el uso de herramientas pesadas.

Existen dos etiologías básicas en las fracturas mandibulares (excluyendo las fracturas patológicas) en trauma. Estas son los accidentes de tránsito y las agresiones (Fridrich et al.; Lin et al.). En este estudio se suma una tercera causa que son los golpes con objeto (herramienta o material industrial). Es conocido que las causas de las fracturas varían su incidencia dependiendo de las características geográficas, sociales y económicas del lugar donde se estudie. En este caso, Chile con altas tasas de construcción, provoca este tipo de fracturas en labores de edificación, probablemente por una falta en las medidas de prevención en labores de riesgo.

La relación entre causa y localización mostrado por los datos en este estudio revelan que las agresiones tienden al igual que el golpe con objeto a producir fracturas parasinfisiarias y de cuerpo junto con la zona condilar (en algunos casos ambos). Una vez resultado el golpe contra la mandíbula, esta se fractura a nivel directo donde recibe el golpe o se transmite la fuerza hacia el cóndilo, debido a su arco con zonas de menor resistencia a nivel del cuello del cóndilo y zona del nervio mental. A diferencia de los accidentes por motocicleta que causan más fracturas a nivel condilar.

Veintidós pacientes $(29,7 \%)$ sufrieron una fractura mandibular múltiple. Dieciséis con dos rasgos y 6 triple rasgo de fractura. Por las características de requerir un trauma de mayor energía, tienden a ocurrir en accidentes de tránsito. Doce pacientes de este grupo presentaron fracturas combinadas contralaterales y 6 pacientes fracturas ipsilaterales, siendo estas últimas de mayor dificultad quirúrgica. Solo 3 pacientes presentaron fractura bicondilea junto con un rasgo anterior.
En cuanto a la tasa de complicaciones (8\%), fue similar a los datos de otros estudios con uso de fijación interna rígida (Fijación interna funcional hoy en día) (Holt, 1986; Fridrich et al.). Todas las etiologías en los pacientes que presentaron una complicación, fueron distintas. Al parecer en este grupo las complicaciones son más relacionadas al tipo de tratamiento, ya que en todas se usó elementos de osteosíntesis. 4 de 6 pacientes tenían directa relación con la oclusión, en una población donde el estado periodontal es malo (lida et al., 2001), es esperable que puedan presentar alteraciones del tipo oclusal y/o articular, en una mordida previamente inestable.

El tratamiento de elección es la reducción abierta y elementos de osteosíntesis con sistema 2.0 o su equivalente 0.8 en sistema Matrix Mandibular Synthes ${ }^{\circledR}$. Para esto se usaron principalmente accesos intraorales, en algunos casos se requirió acceso extraoral, principalmente para acceder al cóndilo. En cuanto a las fracturas múltiples, se ha visto una mayor tasa de complicaciones en las ipsilaterales (Oikarinen et al., 1993), lo que no se repitió en este estudio. En cuanto a las bilaterales se trata en primera instancia, en su mayoría, el área participante en la oclusión dentaria. El uso del sistema Lag Screw es usado en pocas oportunidades, al no tener un protocolo estricto de tratamiento queda sujeto a la elección del cirujano.

Este análisis revela el patrón de las fracturas mandibulares en la población chilena trabajadora. Sin embargo, este estudio presenta varias limitaciones. En Santiago existen tres hospitales aseguradores de los trabajadores. Todos los hospitales en Chile (públicos, privados y hospitales mutuales) tratan pacientes con fracturas faciales, sin embargo algunos reciben más pacientes que otros, quedando fuera de éste muestreo estas dos otras entidades. Los accidentes laborales son tratados por estas mutuales y los accidentes dependientes de consumo de alcohol y traumas no ligados al trabajo en los públicos y privados. Por lo que es cuestionable si los resultados de nuestro estudio pueden ser homologables a toda la población de Santiago, por esta razón, son requeridos los estudios multicéntricos.

Además, al igual que otros estudios retrospectivos, este estudio descriptivo retrospectivo puede estar sujeto a sesgos de información. Sin embargo, estos resultados que se presentan están en línea con otros estudios y el análisis de éste reporte provee de información importante hacia donde enfocar el diseño de planes de prevención de daño, especialmente sobre las medidas del desplazamiento del tránsito y faenas durante labores de construcción. 
GONZALEZ, M. L. E.; VARGAS, F. I.; PEDEMONTE, T. C.; VERDUGO-AVELLO, F.; CANALES, T. M. \& SÁEZ, S. F. Analysis of mandibular fractures caused by work related accidents. Retrospective descriptive study. Int. J. Odontostomat., 9(2):198-203, 2015.

ABSTRACT: The objective of this study was to evaluate the tendency of patients with mandibular fractures associated with working accidents. A retrospective descriptive study was performed in all cases of mandibular fractures who attended the maxillofacial surgery service at the Clinical Hospital Mutual Seguridad C.CH.C. Santiago de Chile, in a 4-year period (January 2009 - December 2012). All mandibular fractures were recorded. The information was collected through review of electronic medical records. Between 2009 and 2012, 74 patients were included in the study with 102 lines of mandibular fractures. The most common fracture was the mandibular condyle (35 patients). The complication rate was $8 \%$. The results shown in this study are in line with the literature and the analysis of this report provides information for the design of risk prevention plans, especially in developing measures of facial protection.

\section{KEY WORDS: mandibular fracture, work related accident, retrospective study.}

\section{REFERENCIAS BIBLIOGRÁFICAS}

Bataineh, A. B. Etiology and incidence of maxillofacial fractures in the north of Jordan. Oral Surg. Oral Med. Oral Pathol. Oral Radiol. Endod., 86(1):31-5, 1998.

Boole, J. R.; Holtel, M.; Amoroso, P. \& Yore, M. 5196 mandible fractures among 4381 active duty army soldiers, 1980 to 1998. Laryngoscope, 111(10):1691-6, 2001.

Bormann, K. H.; Wild, S.; Gellrich, N. C.; Kokemüller, H.; Stühmer, C.; Schmelzeisen, R. \& Schön, R. Five-year retrospective study of mandibular fractures in Freiburg, Germany: incidence, etiology, treatment, and complications. J. Oral Maxillofac. Surg., 67(6):1251-5, 2009.

Edwards, T. J.; David, J. D.; Simpson, D. A. \& Abbott, A. A. Patterns of mandibular fractures in Adelaide, South Australia. Aust. N. Z. J. Surg., 64(5):307-11, 1994.

Ellis, E. 3rd.; Moos, K. F. \& el-Attar, A. Ten years of mandibular fractures: an analysis of 2,137 cases. Oral Surg. Oral Med. Oral Pathol., 59(2):120-9, 1985.

Fridrich, K. L.; Pena-Velasco, G. \& Olson, R. A. Changing trends with mandibular fractures: a review of 1,067 cases. J. Oral Maxillofac. Surg., 50(6):586-9, 1992.

Gamonal, J.; Mendoza, C.; Espinoza, I.; Muñoz, A.; Urzúa, I.; Aranda, W.; Carvajal, P. \& Arteaga, O. Clinical attachment loss in Chilean adult population: First Chilean National Dental Examination Survey. J. Periodontol., 81(10):1403-10, 2010.

Girotto, J. A.; MacKenzie, E.; Fowler, C.; Redett, R.; Robertson, B. \& Manson, P. N. Long-term physical impairment and functional outcomes after complex facial fractures. Plast. Reconstr. Surg., 108(2):312-27, 2001.

Hogg, N. J.; Stewart, T. C.; Armstrong, J. E. \& Girotti, M. J. Epidemiology of maxillofacial injuries at trauma hospitals in Ontario, Canada, between 1992 and 1997. J. Trauma, 49(3):42532, 2000.

Holt, R. G. Maxillofacial Trauma. In: Cummings, C. W.; Frederickson, J. M.; Harker, L. A.; Krause, C. J. \& Schuller, D. E. Otolaryngology-Head and Neck Surgery. St. Louis, Mosby Company, 1986. pp. 313-4. lida, S.; Kogo, M.; Sugiura, T.; Mima, T. \& Matsuya, T. Retrospective analysis of 1502 patients with facial fractures. Int. J. Oral Maxillofac. Surg., 30(4):286-90, 2001.

Lamphier, J.; Ziccardi, V.; Ruvo, A. \& Janel, M. Complications of mandibular fractures in an urban teaching center. J. Oral Maxillofac. Surg., 61(7):745-9, 2003.

Lin, S.; Levin, L.; Goldman, S. \& Peled, M. Dento-alveolar and maxillofacial injuries - a retrospective study from a level 1 trauma center in Israel. Dent. Traumatol., 23(3):155-7, 2007.

Oikarinen, K.; Ignatius, E.; Kauppi, H. \& Silvennoinen, U. Mandibular fractures in northern Finland in the 1980s--a 10-year study. Br. J. Oral Maxillofac. Surg., 31(1):23-7, 1993.

Patrocínio, L. G.; Patrocínio, J. A.; Borba, B. H.; Bonatti, Bde. S.; Pinto, L. F.; Vieira, J. V. \& Costa, J. M. Mandibular fracture: analysis of 293 patients treated in the Hospital of Clinics, Federal University of Uberlândia. Braz. J. Otorhinolaryngol., 71(5):560-5, 2005.

Timoney, N.; Saiveau, M.; Pinsolle, J. \& Shepherd, J. A comparative study of maxillo-facial trauma in Bristol and Bordeaux. J. Craniomaxillofac. Surg.,18(4):154-7, 1990.

Vetter, J. D.; Topazian, R. G.; Goldberg, M. H. \& Smith, D. G. Facial fractures occurring in a medium-sized metropolitan area: recent trends. Int. J. Oral Maxillofac. Surg., 20(4):214-6, 1991.

Dirección para Correspondencia:

Cátedra y Clínica Cirugía y Traumatología Maxilofacial

Facultad de Odontología, Sede Concepción

Universidad Andrés Bello

Concepción

CHILE

Email: verdugo_fco@hotmail.com 